\title{
Realizing the full potential of precision health: The need to include patient-reported health behavior, mental health, social determinants, and patient preferences data
}

\author{
Russell E. Glasgow ${ }^{1,2,3^{*}}$, Bethany M. Kwan ${ }^{1,2}$ and Daniel D. Matlock ${ }^{2,3,4}$ \\ ' Department of Family Medicine, University of Colorado School of Medicine, Aurora, CO, USA \\ ${ }^{2}$ Dissemination and Implementation Science Program of ACCORDS (Adult and Child Consortium for Health Outcomes Research and Delivery Science, Aurora, CO, USA \\ ${ }^{3}$ VA Eastern Colorado Geriatric Research Education and Clinical Center, Denver, CO, USA \\ ${ }^{4}$ Department of Medicine, University of Colorado School of Medicine, Aurora, CO, USA
}

Journal of Clinical and Translational Science (2018), 2, pp. 183-185 doi:10.1017/cts.2018.31

Precision health and big data approaches have great potential, yet such benefits will be realized only when social and behavioral determinants of health and patient preferences are combined with genomic information. Literature review and co-author experiences informed this commentary. Validated health behavior, mental health, and patient preference measures were collected and summarized in real time. Integration of such data into existing data sets will advance precision health, patient-centered care, research, and policy.

Received 30 January 2018; Revised I May 2018; Accepted I6 May 2018, First published online 13 September 2018

Key words: Precision medicine, social and behavioral data, electronic health records, patient-centered care, learning health care system.

\section{Introduction}

The 2 I st Century Cures Act promises to advance the important and rapidly growing science of precision medicine [I]. Chambers et al. [2] recently discussed the potential benefits of integrating advances in precision health and implementation science with learning health care systems. They argue that combining these content areas can improve both individual and public health through enhanced personalization of care, based on "integration of genomics and other precision medicine interventions within real-world practice." Relatedly, the Precision Medicine Initiative is intended to support personalized health care decision making using "genomic, biological, behavioral, environmental, and other data on individuals" [3]. However, the emphasis on and relative clarity around the value of genomic and biological data for

* Address for correspondence: R. E. Glasgow, PhD, Department of Family Medicine, University of Colorado, 1263I E. 17th Avenue, AOI, \#342I, Aurora, CO 80045, USA. (Email: Russell.glasgow@ucdenver.edu) personalized care has resulted in these types of data dominating investments in precision medicine to date [4] - such as those in the 2 Ist Century Cures Act [I].

Truly personalized care will be fully realized only when we also include and investigate the separate and combined impact of the aforementioned "other data" italicized above within precision health interventions. From implementation science and learning health care system perspectives, such data will ideally be those that take into consideration contextual social and behavioral determinants of health, as well as patient values and preferences; and address the logistics of data collection, analysis and use in real-world practice [5]. There are 4 key types of information that could readily be added to current precision health databases to enhance personalization of care. These vital types of data include patient health behaviors; mental and behavioral health; social, economic, and cultural determinants of health; and patient preferences. We briefly discuss each of these factors that have individually been shown to be determinants of individual and public health. When combined with biological information, these additional data have enormous potential to improve health and inform care. They should also enhance predictive science and health services research, given the well-documented interactions between and among these factors and biologic determinants. 
Health behaviors such as smoking, sedentary behavior, poor eating patterns, nonadherence to medication regimens, and risky sexual behavior are strongly associated with increased risk of morbidity (cardiovascular disease, cancer, diabetes) and mortality [6]. This risk decreases substantially following evidence-based individual and public health behavior change interventions [7, 8]. Thus, there is an urgent need to assess these behaviors as part of learning health care systems and to target evidence-based health behavior change interventions tailored to appropriate patients.

Mental and behavioral health issues-depression, anxiety, alcohol and substance use, stress and disease distress-are also key determinants of health. They are common comorbidities with chronic disease, known to exacerbate those conditions and worsen outcomes [9]. It is now a care guideline and accepted best practice to assess depression symptoms, and use these assessment data to direct treatment or refer patients to mental health services (https://www.uspreventiveservices taskforce.org/Page/Document/RecommendationStatementFinal/depress ion-in-adults-screening). Extending this practice to other mental and behavioral health concerns could increase access to services and help to prevent and manage many conditions. But, such activities are only possible if there are efficient, practical, rapid ways to assess and provide actionable feedback on these issues to both patients/families and health care teams. In addition, precision health approaches can help determine which therapies will work for which patients. For example, an interaction between genetic information and depression or physical activity may help tailor the most appropriate combination of therapeutic medications and psychosocial interventions to those patients most likely to benefit.

The frequently cited quip that one's health status is better predicted by one's zip code than by their genetic code has some truth and empirical support given the current state of science, especially for our most prevalent chronic illnesses and complex conditions [10]. Emerging data demonstrate that environmental factors and key "social determinants of health" issues [ I I] such as exposure to violence, food scarcity, poverty, lack of housing, as well as race, ethnic, gender, education, health literacy and numeracy are powerful determinants and comorbid issues for many conditions [ I I ]. Innovations linking biomedical data with these social and behavioral variables will contribute a wealth of research and clinical data regarding how to achieve the greatest improvements in health for individuals and populations.

"Full" precision medicine is not just biological. The importance of patient values and preferences has become increasingly appreciated as a pillar of quality health care [4]. Indeed, the establishment of agencies like the Patient Centered Outcomes Research Institute and the inclusion of mandates for shared decision making in recent Medicare coverage decisions are reflections of our ethical mandate to assure that patients' goals and values drive decisions [12].

Finally, there is an interactive or multiplicative effect of combining these risk factors and data sources (health behavior plus mental health plus social determinants plus patient preferences) with genomic, biomarker, diagnostic and pharmacy data. Collecting and rapidly summarizing key results on these factors can help patients and providers to make more informed, individualized decisions about health care options.

There have been rapid advances in precision health and parallel recent advances in feasible, patient-reported measures of the 4 factors above that result in reliable, valid, and actionable data [13-15]. The $\mathrm{NIH}$ and other funders have made investments in efficient methods and measures to quantify patient status on these factors (www.nihpromis. com). These data can now be collected rapidly, very efficiently and at extremely low cost, and assessments that can provide real-time feedback are in the public domain [15]. Recent research has documented that relevant, pragmatic, patient report assessments of the above domains can produce rapid, actionable results $[16,17]$.
Until recently, patient-reported measures have been regarded as unscientific (when feasible to administer) or too lengthy, impractical, or academic to use in real-world health care settings (when rigorous and validated). Recent research has, however, demonstrated that wellvalidated measures of health behavior, mental health, and patient preference information can be collected efficiently and results rapidly summarized and fed back to both patients/families and health care teams [15]. Such feasibility data have been collected across diverse settings, including low-resource health systems such as community health centers, with both English and Spanish speaking and low income and low literacy patient populations. Data have been collected and results provided in ways tailored to individual clinic and patient situations and preferences (e.g., via internet or phone calls prior to a visit; in the exam room or at the beginning of a clinic visit using computer tablets, or having items read to low literacy patients; results printed out or entered into the electronic health record) $[15,18]$.

Admittedly, there are challenges to incorporating these patientreported data into the electronic health record in an efficient and Health Insurance Portability and Accountability Act-compliant manner, but these have been solved in pilot projects and for related topics $[14,17,18]$, and with the adoption of Fast Healthcare Interoperability Resources [19] standards should become much easier across platforms. Case studies and experiments are needed to show that incorporation of social, behavioral, and patient preferences, and social determinants of health factors, combined with genomics and biologic data, produce superior personalized recommendations, more patientcentered decisions, and enhanced outcomes compared with personalization based on genomic and biologic data alone.

Other directions for future research include: (I) investigation of the settings, patient populations, clinical and community contexts, and conditions under which these patient centered measures are most and least practical and cost-effective; (2) study of the level of training, support, and resources needed for both patients and staff to make optimal use of these new tools and data; and (3) integration of the types of data discussed in this article with real-time, patient-generated data from telemonitoring, digital health tools, and wearable devices. This would allow for personalized "just-in-time" interventions in response to changes in health status-such as adjustments in medications or reminders to follow-up with a care provider or refill prescriptions.

In summary, valid reliable, practical, and actionable patient report measures of health behavior, mental health, patient preferences, and social determinants of health are available that could expand the range of useful data available to construct more fully informed personalized treatment plans. Existing survey measures do have limitations that could be addressed by qualitative approaches to more fully inform and provide contextual information useful for truly personalized care. Qualitative information could be added by strategies such as branching logic to follow-up on key responses with open-ended questions or natural language processing of responses, which could reduce respondent burden. In the future we will also see greater use of computer-adaptive testing [20] to more fully understand patient responses and reduce the number of survey items that patients need to answer even further.

Opportunities to achieve the full potential of precision health as described above will require changes in practice and possibly expansion of current research priorities. What is needed is the political will and commitment to provide patient-centered, personalized care, informed by the best combination of genomic, biological, behavioral, and socialenvironmental information, and to create an infrastructure that supports and values these issues. This integration is achievable but will require a significant investment driven by a broader conceptualization of precision health and new funding priorities. Such an investment should produce substantial dividends in precision health through improved prediction, care management, and patient-centered care. 


\section{Financial Support}

Dr. Glasgow's work on this paper was partially supported by NHLBI KI2 Training grant no. KI2HLI37862 and both his and Dr. Matlock's work partially supported by the Eastern Colorado Healthcare System Veterans Health Administration GRECC award.

\section{Acknowledgments}

The authors wish to acknowledge Linda Niebauer for her assistance with preparation, submission and revision of the manuscript.

\section{Disclosures}

The authors have no conflicts of interest to declare.

\section{References}

I. II4th Congress (2015-2016). 2Ist Century Cures Act. H.R. 342015.

2. Chambers DA, Feero WG, Khoury MJ. Convergence of implementation science, precision medicine, and the learning health care system: a new model for biomedical research. Journal of American Medical Association 2016; 315: 1941-1942.

3. Printz C. Precision medicine initiative boosts funding for $\mathrm{NCl}$ efforts: proposal would help broaden availability of targeted therapies. Cancer 2015; 121: 3369-3370.

4. Institute of Medicine (U.S.), et al. (eds.). Genomics-Enabled Learning Health Care Systems: Gathering and Using Genomic Information to Improve Patient Care and Research: Workshop Summary. Washington, DC: National Academies Press, 2015.

5. Pickett KE, Wilkinson RG. Income inequality and health: a causal review. Social Science \& Medicine 2015; 128: 316-326.

6. Fisher EB, et al. Behavior matters. American Journal of Preventive Medicine 20I I; 40: el5-e30.

7. Anthonisen NR, et al. The effects of a smoking cessation intervention on 14.5-year mortality: a randomized clinical trial. Annals of Internal Medicine 2005; 142: 233-239.
8. Kahn EB, et al. The effectiveness of interventions to increase physical activity. A systematic review. American Journal of Preventive Medicine 2002; 22: 73-107.

9. Chesney E, Goodwin GM, Fazel S. Risks of all-cause and suicide mortality in mental disorders: a meta-review. World Psychiatry 2014; 13 : 153-160.

10. Slade-Sawyer $\mathbf{P}$. Is health determined by genetic code or zip code? Measuring the health of groups and improving population health. North Carolina Medical Journal 2014; 75: 394-397.

II. Marmot M, et al. Commission on Social Determinants of Health. Closing the gap in a generation: health equity through action on the social determinants of health. Lancet 2008; 372: 1661-1669.

12. Barry MJ, Edgman-Levitan S. Shared decision making-pinnacle of patient-centered care. North England Journal of Medicine 2012; 366: 780-78I.

13. Bradley SM, Rumsfeld JS, Ho PM. Incorporating health status in routine care to improve health care value: the VA patient reported health status assessment (PROST) system. Journal of American Medical Association 2016; 316: 487-488.

14. Rabin BA, et al. Advancing the application, quality and harmonization of implementation science measures. Implement Science 20 12; 7: I19.

15. Krist AH, et al. The impact of behavioral and mental health risk assessments on goal setting in primary care. Translational and Behavioral Medicine 2016; 6: 212-219.

16. Riley WT, et al. Rapid, responsive, relevant (R3) research: a call for a rapid learning health research enterprise. Clinical and Translational Medicine 2013; 2: 10.

17. Glasgow RE, Riley WT. Pragmatic measures: what they are and why we need them. American Journal of Preventive Medicine 2013; 45: 237-243.

18. Krist AH, et al. Adoption, reach, implementation, and maintenance of a behavioral and mental health assessment in primary care. Annals of Family Medicine 2014; 12: 525-533.

19. Alterovitz G, et al. SMART on FHIR genomics: facilitating standardized clinico-genomic apps. Journal of the American Medical Informatics Association 2015; 22: 1173-1178.

20. Loe BS, Stillwell D, Gibbons C. Computerized adaptive testing provides reliable and efficient depression measurement using the CES-D scale. Journal of Medical Internet Research 2017; 19: e302. 